\title{
Smaller Than Expected Benefit of COVID-19 Lockdown on Urban Air Quality
}

\author{
Zongbo Shi \\ University of Birmingham, UK \\ z.shi@bham.ac.uk
}

\begin{abstract}
The COVID-19 lockdowns led to major reductions in air pollutant emissions. Here, we quantitatively evaluate changes in ambient $\mathrm{NO} 2, \mathrm{O} 3$, and PM2.5 concentrations arising from these emission changes in 11 cities globally by applying a deweathering machine learning technique. Sudden decreases in deweathered NO2 concentrations and increases in O3 were observed in almost all cities. However, the decline in NO2 concentrations attributable to the lockdowns was not as large as expected, at reductions of 10 to $50 \%$. Accordingly, $\mathrm{O} 3$ increased by 2 to $30 \%$ (except for London), the total gaseous oxidant (Ox = NO2 + O3) showed limited change, and PM2.5 concentrations decreased in most cities studied but increased in London and Paris. Our results demonstrate the need for a sophisticated analysis to quantify air quality impacts of interventions and indicate that true air quality improvements were notably more limited than some earlier reports or observational data suggested.
\end{abstract}

